\title{
FIRST EXCESS LEVELS OF VECTOR PROCESSES
}

\author{
JEWGENI H. DSHALALOW \\ Department of Applied Mathematics \\ Florida Institute of Technology \\ Melbourne, FL 32901, U.S.A.
}

(Received April, 1994; revised August, 1994)

\begin{abstract}
This paper analyzes the behavior of a point process marked by a two-dimensional renewal process with dependent components about some fixed (two-dimensional) level. The compound process evolves until one of its marks hits (i.e. reaches or exceeds) its associated level for the first time. The author targets a joint transformation of the first excess level, first passage time, and the index of the point process which labels the first passage time. The cases when both marks are either discrete or continuous or mixed are treated. For each of them, an explicit and compact formula is derived. Various applications to stochastic models are discussed.
\end{abstract}

Key words: Fluctuations, two-dimensional marked renewal process, firstpassage time, first excess level, termination index, queueing, quorum, vacations, N-policy, T-policy.

AMS (MOS) subject classifications: Primary 60K10; Secondary 60K15, $60 \mathrm{~K} 25$.

\section{Introduction}

In this paper the author studies the behavior of a two-dimensional compound renewal process (with dependent components) about some fixed two-dimensional level. The process will evolve until one of the components hits (i.e. reaches or exceeds) its assigned level for the first time. The following associated random variables are considered: the first passage time, the first excess level (i.e. the value of the process at the first passage time) and the termination index.

These and similar problems belong to the Fluctuations of Stochastic Processes that was extensively studied by many $[1,7,8,12,17,20,22-28]$. The most significant contributions to this theory were made by Lajos Takács who studied behaviors of stochastic processes more general than renewal processes (such as recurrent and semi-Markov processes). In his widely referred-to works [23-28], Takács extended prominent results of his predecessors, Andersen [7], Baxter [8], Pollaczek [20], Spitzer [22] and others. He applied sophisticated analytic techniques to study general fluctuations phenomena by solving relevant recurrent operator equations in terms of operators acting in classes of Banach algebras. The latter was formalized and described by him in [28]. (For more details see also [13] in this issue.) Takács' results were so fundamental and comprehensive that they seemed to deter many to continue his studies on fluctuations, which may have contributed to some slow down of interest in this topic in the eighties. The author of this paper felt inspired rather than intimidated by Takács' impressive results, and was drawn to much smaller but still 
worthy problems.

The problem studied in this paper was motivated by a class of stochastic models in which systems alter their modes as soon as their input processes hit certain specified levels. In Abolnikov and Dshalalow [1] and Dshalalow [12] the authors considered various problems, where a renewal process, observed at random times, hits a fixed level. Such a phenomenon takes place in power stations, stock markets, and it occurs in queueing systems with quorum (Abolnikov and Dshalalow [2,3], Abolnikov, Dshalalow and Dukhovny [5], Dshalalow [10,11] and Dshalalow and Tadj $[14,15]$ ), N-policy (Muh [19]), T-policy (Heyman [18] and Shellhaas [21]), and with vacationing servers (Abolnikov and Dshalalow [4], Abolnikov, Dshalalow and Dukhovny [6], Dshalalow [9] and Dshalalow and Yellen [16]). For instance, in a fairly general multiple-vacation queue, a server (or servers) begins its vacationing sequence when, upon completion of a service (frequently - group service), the queue drops below level $r$. The sequence of vacations is terminated when the queue accumulates to level $R(\geq r)$. The queue lengths observed at the end of each vacation segment can serve as such a compound process which hits level $R$. (Such a system was introduced and studied in Dshalalow and Yellen [16].)

In contrast to this and similar scenarios, a targeted queueing process may have more than one random component, and this unorthodox modification of the above models may be of practical interest. The hitting time or first passage time of such a multi-dimensional process will be the first instant of time when one of the components of the process reaches or exceeds its associated level. An appropriate example may come again from queueing, where a single server rests or goes on vacation, whenever the queue drops below $r$. A service will be restored when the queue hits $R$ or the cumulative job (expressed as the total service time needed to process all customers in the queue) hits $S$, whichever comes first.

Another example of such a process is a queueing system with "two-dimensional arrivals." The first component of each arrival may represent the batch size (number of customers in that arrival) and the second component might be another random variable associated with that batch of customers (e.g. customers' baggage, cumulative account balance, investment or debt, amount of fluid, etc.). Here the server also rests or goes on vacations, and then resumes service as soon as the total number of customers is at least $R$ or the associated cumulative value of the second component hits $S$. The analysis of such systems would undoubtedly be useful in many applications.

In [12] the author studied a compound delayed renewal process observed at random moments of time $\tau_{0}, \tau_{1}, \ldots$ that was terminated by either hitting a fixed level (at one of these moments) or possibly earlier at $\tau_{\sigma}$, where $\sigma$ is an arbitrarily distributed integer-valued random index, independent of the process. The problem proposed in this paper formally generalizes the one studied in [12]. Here the compound renewal process has a second (generally dependent) mark. In the scenario of [12], the second mark is taken discrete with increments equal one a.s. over $\tau_{n}-\tau_{n-1}$, $n=1,2, \ldots$, and it is assumed to be independent of the first ("principal") mark. Then, the problem studied here will reduce to that in [12], if we modify the level, which the second mark is supposed to reach, by making it random. [The present paper does not discuss how to do this though.]

The author targets joint transformation of the first excess level, first passage time, and the index $\Delta$ of the point process $\left\{\tau_{n}\right\}$ which labels the first passage time $\tau_{\Delta}$. The cases, when both marks are either discrete or continuous or mixed, are distinguished. In all of them, explicit and compact formulas are obtained. Some applications to queueing with quorum and vacationing server are discussed. Also, new queueing systems with "two-dimensional" arrivals are proposed. 


\section{Formal Description}

All random variables and stochastic processes throughout this paper will be considered on a probability space $\{\Omega, \mathscr{F}, P\}$. Let $Z=\sum_{n \geq 0}\left(X_{n}, Y_{n}\right) \varepsilon_{\tau_{n}}$ (where $\varepsilon_{a}$ is a point mass) be a marked random counting measure with the following assumptions. The random vectors $\left(X_{n}, Y_{n}\right)$, $n=0,1, \ldots$, are valued in $\mathbb{R}_{+} \times \mathbb{R}_{+}$, independent, and for $n \geq 1$ identically distributed. [Note that for each vector $\left(X_{n}, Y_{n}\right)$, its components need not be independent.] The counting measures, $\tau=\sum_{n \geq 0} \varepsilon_{\tau_{n}}$ on $\left(\mathbb{R}_{+}, \mathfrak{B}\left(\mathbb{R}_{+}\right)\right.$) (where $\mathfrak{B}$ is the Borel $\sigma$-algebra generated by the usual topology on the real line) and $(A, B)=\sum_{n=0}^{\infty} \varepsilon_{\left(A_{n}, B_{n}\right)}$ on $(\Psi, \mathfrak{B}(\Psi)), \Psi \subseteq \mathbb{R}_{+}$, are delayed renewal processes, and $Z$ is obtained from $\tau$ by position independent marking. Consequently, $(A, B)=\left(A_{n}=\sum_{k=0}^{n} X_{k}, B_{n}=\sum_{k=0}^{n} Y_{k} ; n=0,1, \ldots\right)$ is a two-dimensional delayed renewal process. Let

$$
a_{0}(u, v)=\mathbf{E}\left[u^{X_{0}} v^{Y} 0\right], a(u, v)=\mathbf{E}\left[u^{X_{1}} v^{Y_{1}}\right], h_{0}(\theta)=\mathbf{E}\left[e^{-\theta \tau_{0}}\right], h(\theta)=\mathbf{E}\left[e^{-\theta\left(\tau_{1}-\tau_{0}\right)}\right]
$$

Let $p_{1}$ and $p_{2}$ be two real non-negative numbers designed as two levels, one for $(A)$ and the second for $(B)$ to reach or exceed these numbers for the first time. We will say that $(A, B)$ hits level $L=\left(p_{1}, p_{2}\right)$, or, equivalently, $L$ is the first excess level for $(A, B)$ if for some $n, A_{n}$ or $B_{n}$ reach or exceed at least one of their respective levels, $p_{1}$ or $p_{2}$, for the first time. The "first passage time" is placed on the same axis as $\tau$, and it will denote the instant of time when $(A, B)$ hits $L$. More formally, if

$$
\nu_{1}=\nu_{1}\left(p_{1}\right)=\inf \left\{k: A_{k} \geq p_{1}\right\} \text { and } \nu_{2}=\nu_{2}\left(p_{2}\right)=\inf \left\{k: B_{k} \geq p_{2}\right\}
$$

and

$$
\Delta=\min \left\{\nu_{1}, \nu_{2}\right\}
$$

which is the index of the first excess of $L$ by $(A, B)$ or just the termination index, then $\tau_{\Delta}$ is the first passage time when $(A, B)$ hits $L$. Consequently, $\left(A_{\Delta}, B_{\Delta}\right)$ is the value of the first excess of level $L$.

\section{Preliminaries}

Let $f$ and $g$ be integrable functions with respect to relevant Borel measures and weight functions. Consider two operators which we for convenience denote by one symbol, $D_{p}$, and we will distinguish them by calling them types $a$ and $b$ :

$$
\begin{gathered}
D_{p}(f(p))(z)=(1-z) \sum_{p \geq 0} z^{p} f(p),|z|<1, \\
D_{p}(f(p))(s)=s \int_{p=0}^{\infty} e^{-s p} f(p) d p, \operatorname{Re}(s)>0 .
\end{gathered}
$$

Let

$$
D_{p_{1}, p_{1}}\left(g\left(p_{1}, p_{2}\right)\right)(x, y)=D_{p_{1}}\left\{D_{p_{2}}\left(g\left(p_{1}, p_{2}\right)\right\}(x, y),\right.
$$

where on the right-hand side of (3.1) the operators may be of types $a$ or $b$ or mixed. Note that due to Fubini's theorem, the operators on the right-hand side of (3.1) are commutative.

For convenience, we extend the definition of the renewal process $(A, B)$ by setting

$$
A_{-1}=B_{-1}=0 \text {. }
$$

Lemma 1. For the operators of types $a$ and $b$ it holds true that 
and

$$
D_{p_{1}}\left(I_{\left\{\nu_{1}=j\right\}}\right)(x)=x^{A-1}-x^{A} j, j=0,1, \ldots
$$

$$
D_{p_{2}}\left(I_{\left\{\nu_{2}=k\right\}}\right)(y)=y^{B} k-1-y^{B} k, k=0,1, \ldots
$$

Proof. From the definition of $\nu_{1}$, we deduce that

$$
I_{\left\{\nu_{1}=j\right\}}=I_{[0, p]}\left(A_{j-1}\right)-I_{[0, p]}\left(A_{j}\right), j=0,1, \ldots,
$$

where $I_{G}$ is the indicator function of a set $G$. This yields the above assertion for the operators of types $a$ and $b$.

We shall be interested in an analytic representation of

$$
\mathbf{E}\left[\xi^{\Delta} e^{\left.-\theta \tau_{\Delta_{u}}{ }^{A} \Delta_{v}^{B}{ }^{B}\right]}\right.
$$

to which we apply the operator defined in (3.1). We will consider three cases for $(A, B)$ : with both discrete and continuous components and mixed. In the latter case, and this is perhaps the most common scenario in applications, we assume (without loss of generality) that $A$ is a discrete component and $B$ is a continuous component of $(A, B)$.

\section{Discrete Case}

Denote

$$
\mathscr{F}=\mathscr{F}(\xi, \theta, u, v ; x, y)=D_{p_{1}} D_{p_{2}}\left\{\mathbf{E}\left[\xi^{\Delta} e^{-\theta \tau} \Delta_{u}^{A} \Delta_{v}^{B} \Delta\right]\right\}(x, y)
$$

where both operators are of type $a$. Then,

$$
\mathscr{F}=\mathscr{F}_{1}+\mathscr{F}_{2}+\mathscr{F}_{3}
$$

where

and

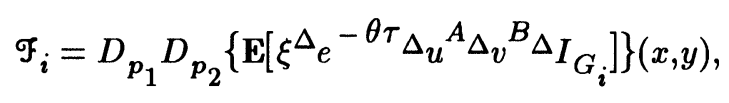

$$
G_{1}=\left\{\nu_{1}<\nu_{2}\right\}, G_{2}=\left\{\nu_{1}=\nu_{2}\right\}, G_{3}=\left\{\nu_{1}>\nu_{2}\right\}
$$

(As before, $I_{G}$ is the indicator function of a set $G$.) Obviously, by Fubini's theorem,

$$
\mathscr{F}_{1}=\sum_{k \geq 1} \sum_{j=0}^{k-1} D_{p_{1}} D_{p_{2}}\left\{\mathbf{E}\left[\xi^{j} e^{-\theta \tau} j_{u}{ }^{A} j_{v}{ }^{B} j_{I_{\left\{\nu_{1}=j\right\}}} I_{\left\{\nu_{2}=k\right\}}\right]\right\}(x, y),
$$

from Lemma 1 and by the independence of $\tau_{j}$ from the rest in $\mathbf{E}$, we have

$$
\left.\mathscr{F}_{1}=h_{0}(\theta) \sum_{k \geq 1} \sum_{j=0}^{k-1}\{\xi h(\theta)\}^{j} \mathbf{E}\left[u^{A} j_{v}{ }^{B} j_{(x}{ }^{A} j-1-x^{A} j\right)\left(y^{B} k-1-y^{B} k\right)\right] .
$$

The last expression can be rewritten as

$$
\mathscr{F}_{1}=h_{0}(\theta) \sum_{k \geq 1} \sum_{j=0}^{k-1}\{\xi h(\theta)\}^{j} E_{1} E_{2}
$$

where $E_{1}$ and $E_{2}$ are the two factors extracted from $\mathcal{F}_{1}$ by using the independent increments property of $(A, B)$ :

$$
E_{1}=\mathbf{E}\left[\left[(u x)^{A} j-1 u^{X}{ }_{-}(u x)^{A} j\right](v y)^{B} j\right], E_{2}=[1-b(y)] b^{k-1-j}(y), \text { with } b(y)=a(1, y) .
$$


After some standard transformations, $E_{1}$ reduces to

$$
E_{1}=\left\{\begin{array}{cc}
a_{0}(u, v y)-a_{0}(u x, v y), & j=0 \\
a_{0}(u x, v y)[a(u, v y)-a(u x, v y)] a^{j-1}(u x, v y), & j>0
\end{array}\right.
$$

and this yields

$$
\frac{1}{h_{0}(\theta)} \mathscr{F}_{1}=a_{0}(u, v y)-a_{0}(u x, v y)+\frac{\xi h(\theta) a_{0}(u x, v y)[a(u, v y)-a(u x, v y)]}{1-\xi h(\theta) a(u x, v y)}
$$

An analogous expression for $\mathcal{F}_{3}$ can be easily deduced from that for $\mathcal{F}_{1}$ by interchanging the roles of the variables $u$ and $x$ with $v$ and $y$ :

$$
\frac{1}{h_{0}(\theta)} \mathcal{F}_{3}=a_{0}(u x, v)-a_{0}(u x, v y)+\frac{\xi h(\theta) a_{0}(u x, v y)[a(u x, v)-a(u x, v y)]}{1-\xi h(\theta) a(u x, v y)}
$$

Now,

$$
\mathcal{F}_{2}=\sum_{k \geq 0} D_{p_{1}} D_{p_{2}}\left\{\mathbf { E } \left[\xi^{k} e^{\left.\left.-\theta \tau_{k} u^{A} k_{v}{ }^{B} I_{\left\{\nu_{1}=\nu_{2}=k\right\}}\right]\right\}(x, y)}\right.\right.
$$

By Lemma $1, \mathcal{F}_{2}$ transforms to

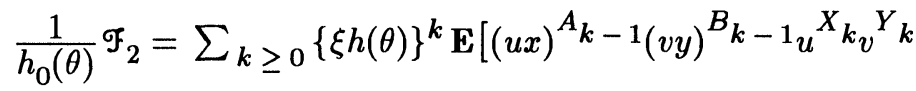

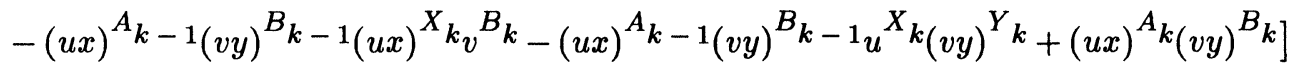

and then further to

$$
\begin{aligned}
& \frac{1}{h_{0}(\theta)} \mathscr{F}_{2}=a_{0}(u, v)-a_{0}(u x, v)+a_{0}(u, v y)-a_{0}(u x, v y) \\
+ & \frac{\xi h(\theta) a_{0}(u x, v y)[a(u, v)-a(u x, v)-a(u, v y)+a(u x, v y)]}{1-\xi h(\theta) a(u x, v y)}
\end{aligned}
$$

Performing the summation of $\mathscr{F}_{1}, \mathscr{F}_{2}$ and $\mathscr{F}_{3}$ we arrive at the following compact formula

$$
\frac{1}{h_{0}(\theta)} \mathscr{F}=a_{0}(u, v)-a_{0}(u x, v y) \frac{1-\xi h(\theta) a(u, v)}{1-\xi h(\theta) a(u x, v y)} .
$$

Define the linear operators:

and

$$
\mathscr{T}_{z}^{k}(\cdot)=\lim _{x \rightarrow 0} \frac{1}{k !} \frac{\partial^{k}}{\partial x^{k}} \frac{1}{1-x}(\cdot)
$$

$$
\oiint_{x, y}^{j, k}(\cdot)=\lim _{(x, y) \rightarrow(0,0)} \frac{1}{j ! k !} \frac{\partial^{k}}{\partial x^{k}} \frac{\partial^{j}}{\partial y^{j}} \frac{1}{(1-x)(1-y)}(\cdot)
$$

Then, taking into consideration $(a)$ and applying the operator $\mathscr{T}_{x, y}^{p_{1}, p_{2}}$ to (4.1) we can restore $\mathbf{E}\left[\xi^{\Delta} e^{-\theta \tau} \Delta_{u}^{A} \Delta_{v}^{B} \Delta_{:}\right.$

$$
\frac{1}{h_{0}(\theta)} \mathbf{E}\left[\xi^{\Delta} e^{-\theta \tau} \Delta_{u}{ }^{A} \Delta_{v}^{B} \Delta^{B}\right]=a_{0}(u, v)-(1-\xi h(\theta) a(u, v)) \mathscr{D}_{x, y}^{p_{1}, p_{2}}\left\{\frac{a_{0}(u x, v y)}{1-\xi h(\theta) a(u x, v y)}\right\}
$$


This generalizes formula (3.4) in Dshalalow [12] which is almost identical to (4.2) but the present case deals with a two-dimensional renewal process. In the event that the components of $(A, B)$ are independent $a_{0}$ and $a$ are factorizable:

$$
a_{0}(u, v)=a_{0}(u) b_{0}(v) \text { and } a(u, v)=a(u) b(v) .
$$

Then we have that

$$
\begin{gathered}
\frac{1}{h_{0}(\theta)} \mathbf{E}\left[\xi^{\Delta} e^{\left.-\theta \tau_{\Delta_{u}}{ }^{A} \Delta_{v}{ }^{B} \Delta\right]}\right. \\
=a_{0}(u) b_{0}(v)-(1-\xi h(\theta) a(u) b(v)) \Phi_{x, y}^{p_{1}, p_{2}}\left\{\frac{a_{0}(u x) b_{0}(v y)}{1-\xi h(\theta) a(u x) b(v y)}\right\}
\end{gathered}
$$

from which we see that $a$ and $b$ appear symmetrically.

\section{Continuous Case}

This refers to the case of the operator $D$ in $(b)$ applied to transformation (3.2), and treats a continuous-valued process $(A, B)$. Only formula (4.1) needs to be considered, since the remaining analysis from section 4 also applies to the continuous case.

With the substitutions $u=e^{-\vartheta_{1}}$ and $v=e^{-\vartheta_{2}}$ we have that

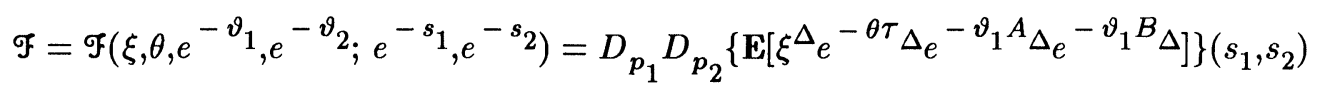

and the "continuous equivalent" of formula (4.1) will then be

$$
\frac{1}{h_{0}(\theta)} \mathscr{F}=\alpha_{0}\left(\vartheta_{1}, \vartheta_{2}\right)-\alpha_{0}\left(\vartheta_{1}+s_{1}, \vartheta_{2}+s_{2}\right) \frac{1-\xi h(\theta) \alpha\left(\vartheta_{1}, \vartheta_{2}\right)}{1-\xi h(\theta) \alpha\left(\vartheta_{1}+s_{1}, \vartheta_{2}+s_{2}\right)}
$$

where

$$
\alpha_{0}\left(\vartheta_{1}, \vartheta_{2}\right)=a_{0}\left(e^{-\vartheta_{1}}, e^{-\vartheta_{2}}\right), \text { and } \alpha\left(\vartheta_{1}, \vartheta_{2}\right)=a\left(e^{-\vartheta_{1}}, e^{-\vartheta_{2}}\right)
$$

\section{Mixed Case}

In this case we assume that mark $A$ be discrete and mark $B$ continuous. A formula analogous to (4.1) and (4.2) will read

$$
\frac{1}{h_{0}(\theta)} \mathscr{F}=\mathcal{A}_{0}\left(u, \vartheta_{2}\right)-\mathcal{A}_{0}\left(u x, \vartheta_{2}+s_{2}\right) \frac{1-\xi h(\theta) \mathcal{A}\left(u, \vartheta_{2}\right)}{1-\xi h(\theta) \mathcal{A}\left(u x, \vartheta_{2}+s_{2}\right)}
$$

with

$$
\mathcal{A}_{0}\left(u, \vartheta_{2}\right)=a_{0}\left(u, e^{-\vartheta_{2}}\right) \text { and } \mathcal{A}\left(u, \vartheta_{2}\right)=a\left(u, e^{-\vartheta_{2}}\right)
$$

This case goes back to the class of queueing models discussed in the introduction. In such a model, a server typically rests or begins its vacations sequence if the queue falls below some level $r$. The vacation sequence is interrupted when the queue once accumulates to at least $p_{1}$ or the cumulative job in the system hits $p_{2}$, whichever comes first. The input to the system may be bulk 
and state dependent. The service may be batch to accommodate more general situations. The second mark, $B$, need not represent the cumulative job. It may be any qualitative component mentioned in the introduction.

Acknowledgement. The author thanks Jay Yellen for his valuable remarks and suggestions.

\section{References}

[1] Abolnikov, L. and Dshalalow, J.H., A first passage problem and its applications to the analysis of a class of stochastic models, Journ. Appl. Math. Stoch. Analysis, 5, No.1 (1992) 83-98.

[2] Abolnikov, L. and Dshalalow, J.H., Ergodicity conditions and invariant probability measure for an embedded Markov chain in a controlled bulk queueing system with a bilevel service delay discipline. Part I, Appl. Math. Letters, 5, No. 4 (1992), 25-27.

[3] Abolnikov, L. and Dshalalow, J.H., Ergodicity conditions and invariant probability measure for an embedded Markov chain in a controlled bulk queueing system with a bilevel service delay discipline. Part II, Appl. Math. Letters, 5, No. 5 (1992), 15-18.

[4] Abolnikov, L., Dshalalow, J.H. and Dukhovny, A.M., A multilevel controlled bulk queueing system with vacationing server, Oper. Res. Letters, 13 (1993), 183-188.

[5] Abolnikov, L., Dshalalow, J.H. and Dukhovny, A.M., First passage processes in queueing system $M^{X} / G^{r} / 1$ with service delay discipline, Intern. Journ. Math. \& Math. Sci., 17, No. 3 (1994), 571-586.

[6] Abolnikov, L., Dshalalow, J.H. and Dukhovny, A.M., Stochastic analysis of a controlled bulk queueing system with continuously operating server: continuous time parameter queueing process, Stat. Prob. Letters, 16 (1993), 121-128.

[7] Andersen, E.S., On the fluctuations of sums of random variables, II, Math. Scand., 2 (1954), 195-223.

[8] Baxter G., On operator identity, Pacific J. Math., 8 (1958), 649-663.

[9] Dshalalow, J.H., On a first passage problem in general queueing systems with multiple vacations, Journ. Appl. Math. Stoch. Analysis, 5, No. 2 (1992), 177-192.

[10] Dshalalow, J.H., Single-server queues with controlled bulk service, random accumulation level, and modulated input, Stoch. Analysis and Appl., 11, No. 1 (1993), 29-41.

[11] Dshalalow, J.H., First excess level analysis of random processes in a class of stochastic servicing systems with global control, Stoch. Analysis and Appl., 12, No. 1 (1994), 75-101.

[12] Dshalalow, J.H., On termination time processes, in Studies in Applied Probability, Edited by J. Galambos and J. Gani, Essays in honor of Lajos Takács, Journ. of Appl. Prob., Special Volume 31A (1994), 325-336.

[13] Dshalalow, J.H. and Syski, R., Lajos Takács and his work, Journ. Appl. Math. Stoch. Anal., 7, No. 3 (1994), 217-238.

[14] Dshalalow, J.H. and Tadj, L., A queueing system with random server capacity and multiple control, Queueing Systems, 14 (1993), 369-384.

[15] Dshalalow, J.H. and Tadj, L., On applications of first excess level random processes to queueing systems with random server capacity and capacity dependent service time, Stoch. and Stoch. Reports, 45 (1993), 45-60.

[16] Dshalalow, J.H. and Yellen, J., On the first excess level analysis of generalized quorum queueing systems with multiple vacations (in preparation).

[17] Dynkin, E., Some limit theorems for sums of independent random variables with infinite mathematical expectations, Sel. Transl. Math. Statist. Prob., 1 (1961), 171-189.

[18] Heyman, D.P., The T-policy for the $M / G / 1$ queue, Manag. Sci., 23 (1977), 775-778. 
[19] Muh, D.C.R., A bulk queueing system under N-policy with bilevel service delay discipline and start-up time, Journ. Appl. Math. Stoch. Analysis, 6, No. 4 (1993), 359-384.

[20] Pollaczek, F. Sur la répartition des périodes d'occupation ininterrompue d'un guichet, C.R. Acad. Sci. Paris 234 (1952), 2042-2044.

[21] Schellhaas, H., On the T-policy for an $M / G / 1$ queue, in Operations Research Verfahren, 29 (1978) 750-763.

[22] Spitzer, F., A combinatorial lemma and its application to probability theory, Trans. Amer. Math. Soc., 82 (1956), 323-339.

[23] Takács, L., On a linear transformation in the theory of probability, Acta Sci. Math., 33, No. 1-2 (1972), 15-24.

[24] Takács, L., Sojourn time problems, Ann. Prob., 2, No. 3 (1974), 420-431.

[25] Takács, L., A storage process with semi-Markov input, Adv. Appl. Prob., 7, No. 4 (1975) 830-844.

[26] Takács, L., On some recurrence equations in a Banach algebra, Acta Sci. Math., 38, No. 34 (1976), 399-416.

[27] Takács, L., On fluctuation problems in the theory of queues, Adv. Appl. Prob., 8, No. 3 (1976) 548-583.

[28] Takács, L., On fluctuations of sums of random variables, Adv. Math, 2 (1978), 45-93. 


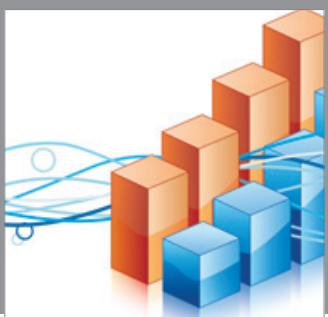

Advances in

Operations Research

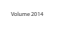

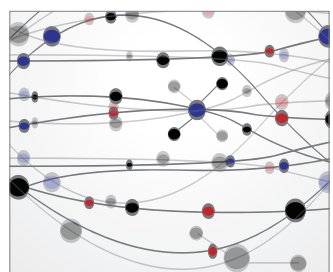

\section{The Scientific} World Journal
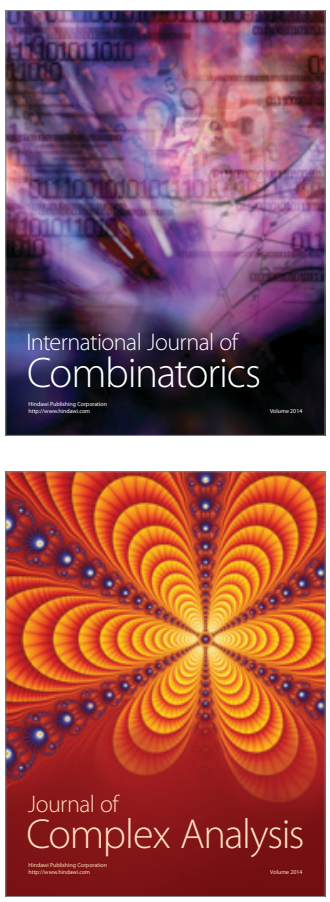

International Journal of

Mathematics and

Mathematical

Sciences
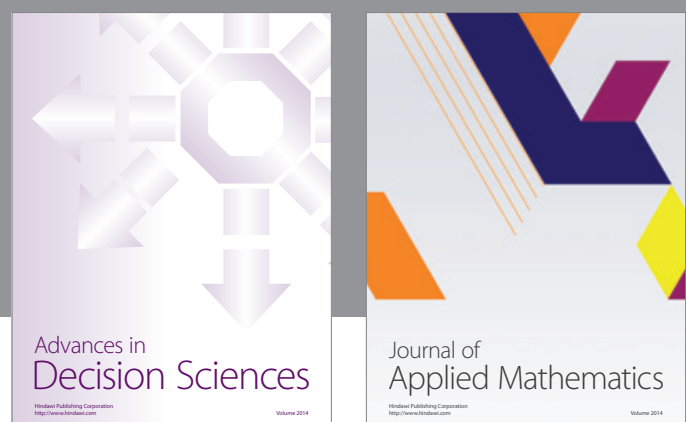

Journal of

Applied Mathematics
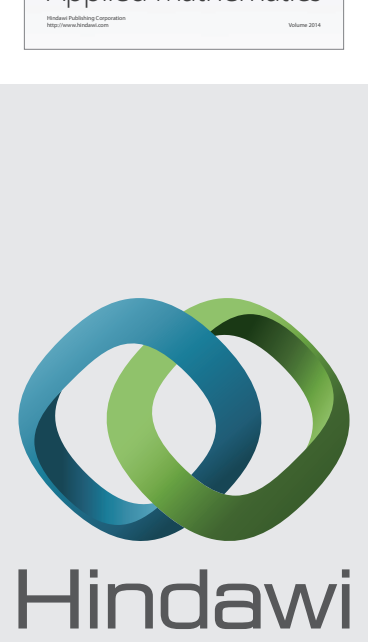

Submit your manuscripts at http://www.hindawi.com
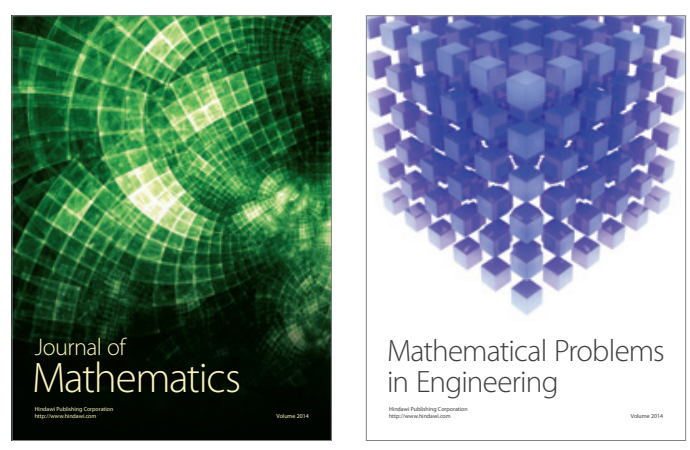

Mathematical Problems in Engineering
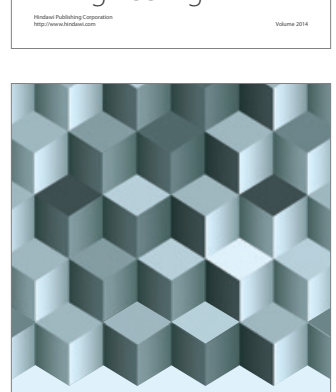

Journal of

Function Spaces
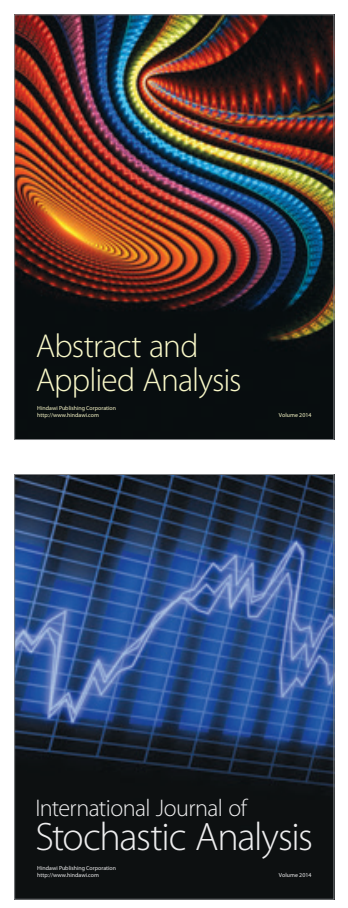

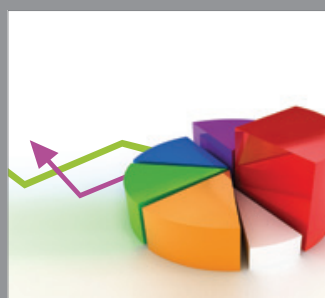

ournal of

Probability and Statistics

Promensencen
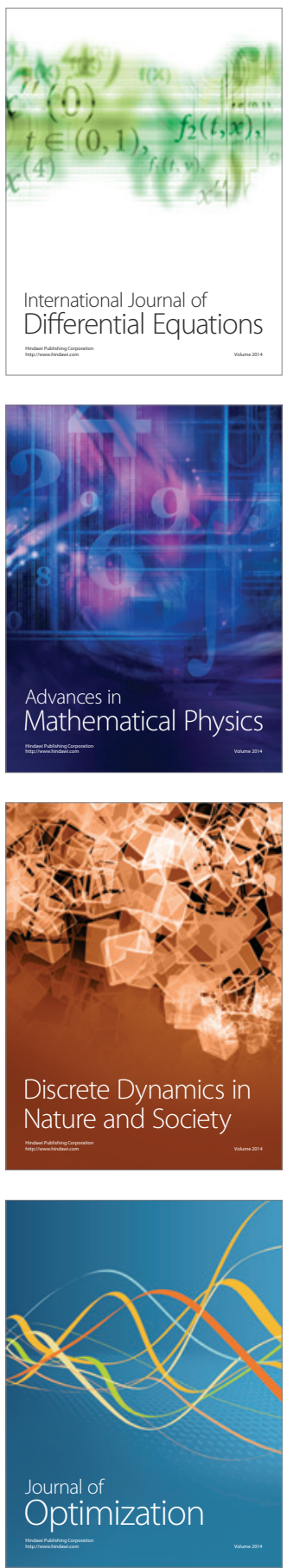